To obtain this information and estimate the relevant source parameters, Lambert and his colleagues have carried out an extremely detailed and lengthy study of the surface wave radiation from two explosions and one earthquake. Their particular aims were, first, to determine from surface wave spectra the differences between the long-period radiation from earthquakes and explosions; second, to determine the spatial radiation patterns from the two types and the differences between them; and, third, to describe the individual sources quantitatively. In a comparative exercise of this kind, there is clearly some advantage in studying at least one earthquake-explosion pair with a common point of origin, common recording stations and, if possible, similar radiated energy. In this way, irrelevant phenomena and complicated corrections can be largely avoided.

The events chosen to conform most closely with these conditions were the nuclear explosion SHOAL $\left(m_{\mathrm{b}}=4.9\right)$ of 1963 and the Fallon earthquake $\left(m_{\mathrm{b}}=4.4\right)$ of 1962 , both of which occurred within $60 \mathrm{~km}$ of each other in Nevada and both of which were recorded by the Long Range Seismic Measurement (LRSM) network. For further comparison between explosions themselves, the BILBY explosion $\left(m_{\mathrm{b}}=5.8\right)$ of 1963 was also investigated. Not only was this event of higher magnitude than SHOAL, it was detonated in tuff (by contrast with granite for SHOAL), thus enabling Lambert et al. to investigate variations with yield and the nature of the source medium.

The important conclusions are, first, that at long periods the surface wave radiation field from the Fallon earthquake is a quadrupole field corresponding to a quadrupole or double-couple source. At higher frequencies, however, the radiation pattern contains asymmetries indicative of multiple contributions of higher order, probably produced by the propagation of ruptures. Both these results agree quantitatively with theoretical predictions. The surface wave radiation from the explosions, on the other hand, is best explained by sources comprising a superimposed monopole and quadrupole. In the frequency range comparable to that over which the earthquake signal was recorded, there was no suggestion of multipole components of higher order. What Lambert and his colleagues regard as their principal conclusion is that the anomalous doublecouple radiation from the explosions is probably not a consequence of earthquake triggering but rather of the relaxation of stress around the shatter zone generated by the shock.

The detailed comparison between the SHOAL explosion and the Fallon earthquake shows, first, that the ratio of the Love wave amplitudes (earthquake/explosion) decreases with increasing frequency. This implies a larger source for the earthquake. The second result is that, whereas for the explosions the normalized spectral ratio of Love wave amplitude to Rayleigh wave amplitude was close to one and almost independent of frequency, the same ratio for the earthquake was two to three times greater and decreased with increasing frequency. In other words, the earthquake generated Love waves far more efficiently than did the explosions and in a manner dependent on frequency. Finally, Lambert et al. thus conclude that these differences might be useful as discriminators between explosions and earthquakes, at least for events with $m_{\mathrm{b}}$ greater than 4.4. On the other hand, as they are careful to point out, there is no guarantee, on the basis of this analysis alone, that all earthquakes behave similarly.

\section{Progress in Gamma-ray Astronomy}

Two contrasting contributions in next Monday's Nature Physical Science (January 22) emphasize the rapid development of gamma-ray astronomy in recent months. In one, Stecker reviews the present status of theory and observation concerning the diffuse cosmic gamma-ray background; in the other, Guthrie and Tademaru put forward a model to explain the presence of a low energy $(473 \pm 30 \mathrm{keV})$ line in the spectrum of gamma rays from the galactic centre.

The observations of Guthrie and Tademaru stem from a balloon flight (Johnson et al., Astrophys. J. Lett., 172, L1; 1972); those of Stecker are chiefly from rocket and satellite experiments, including those on OSO-3 (Kraushaar et al., A strophys. J., 177, 341 ; 1972) and unpublished data obtained during the flight of Apollo 15. The wide range of observational facilities now available is, of course, the underlying reason for the explosion of interest in gamma-ray astronomy, just as in X-ray astronomy.

In the case of the galactic nucleus, Guthrie and Tademaru suggest that a cluster of neutron stars may be generating the observed line through a mechanism in which electron-positron pairs are first produced in the rapidly rotating intense magnetic fields associated with a neutron star and then annihilated at the star's surface. Gravitational redshifting of the resulting radiation could then account for the difference between the observed line energy and the theoretical energy for radiation produced in this way (511 keV). Sturrock's studies of pulsars have shown that sufficient electron-positron pairs might be produced for the mechanism to be effective (Nature, 227, $465 ; 1970$ ), but
RELATIVISTIC ASTROPHYSICS State of the Art

from a Correspondent

ThE Texas Symposia on relativistic astrophysics have become biennial reports on the state of the frontiers of astrophysics. During the most recent, held between December 18 and 22 in New York, some fifty speakers summarized the situation for an audience of more than a thousand astrophysicists and physicists.

The first day was devoted to quasars and radio galaxies. Professor $\mathrm{H}$. Arp (Hale Observatories) opened the session by summarizing the case for a noncosmological origin of redshifts, most of the evidence being based on detailed studies of the unusual members of the extragalactic population. This was followed by several talks given by what could be called anti-Arp forces, who prefer to place emphasis on the normal characteristics of most of this

other suggestions, including the possibility of a nuclear de-excitation line such as that from ${ }^{7} \mathrm{Li}$ (which is stable and has a line energy of $477 \mathrm{keV}$ ), cannot be ruled out in the present state of the art.

Stecker's article is concerned with the cosmological implications of the diffuse gamma rays, rather than with events in our own Galaxy. But again the question of matter-antimatter interactions arises. With the new gamma-ray observations, there is an essentially complete spectrum of the background radiation from $10^{-3}$ to $100 \mathrm{MeV}$. Above $1 \mathrm{MeV}$, the gamma-ray spectrum no longer follows the power law typical of X-ray energies, and the ratio of observed flux to extrapolated flux reaches a maximum of 10 near $20 \mathrm{MeV}$ (see diagram). One way in which additional gamma rays might be produced between 1 and $100 \mathrm{MeV}$ is by matter-antimatter annihilation, with a peak at around 70 $\mathrm{MeV}$ Doppler shifted to lower frequencies by expansion of the universe. Again, there are many other possible interpretations of the data; but this must be one of the most intriguing possibilities, suggesting the presence of large amounts of antimatter in the universe which is able to interact with the matter there.

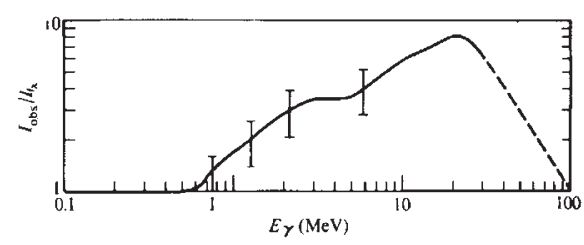

Ratio of observed gamma-ray flux to flux predicted by extrapolation from $\mathrm{X}$-ray energies. 